\title{
Photoperiod regulates the daily profiles of tryptophan hydroxylase-2 gene expression the raphe nuclei of rats \\ ${ }^{1}$ Zeina S. MALEK, ${ }^{2}$ Louay LABBAN
}

\author{
${ }^{I}$ Faculty of Pharmacy, Syrian Private University, Syria. \\ ${ }^{2}$ Faculty of Pharmacy, Al Jazeera Private University, Syria.
}

\begin{abstract}
Tryptophan hydroxylase-2 mRNA (TPH, the rate limiting enzyme in 5-HT synthesis) expression levels display circadian variations in the median and dorsal raphe nuclei. This circadian pattern is under the control of the suprachiasmatic nuclei $(\mathrm{SCN})$, the master clock. Photoperiodic cue is encoded by the SCN which convey the seasonal message to target sites. In the present study, we have investigated the effect of photoperiodic changes on the serotonergic neurons of the raphe nuclei. We have assessed the daily expression of TPH2 mRNA in both median and dorsal raphe nuclei of rats housed either under long photoperiod (18 h light/6 h dark cycle, LP18:6) or short photoperiod (SP6:18). Our results demonstrate that under LP18:6, TPH2 mRNA levels display a progressive decrease during the dark period and a maximal expression is reported at the beginning of the light period. The expression pattern of TPH2 mRNA under SP6:18 remains unchanged during the dark period and increases significantly before the day/night transition. This latter expression pattern is in line with the daily profiles of TPH 2 mRNA reported previously under standard lighting regimen (12 h light/12 h dark cycle). The present results suggest that TPH2 mRNA expression pattern within DR and MR is affected by photoperiod which might in turn affect TPH content and 5-HT release within the circadian structures, but also in all the serotonergic projection areas of the brain.
\end{abstract}

Keywords: Tryptophan hydroxylase-2; suprachiasmatic nuclei; Photoperiodic cue; mRNA.

\section{Introduction}

Serotonin (5-HT) plays an important role in both photic and non-photic resetting of the central circadian pacemaker residing in the suprachiasmatic nuclei $(\mathrm{SCN})$ of the hypothalamus $[1,2]$. A direct serotonergic afferent pathway to the $\mathrm{SCN}$ arises from the median raphe nucleus (MR), whereas the dorsal raphe nucleus (DR) projects indirectly to the SCN through the thalamic intergeniculate leaflets $[3,4]$. Both Tryptophan hydroxylase (TPH, the rate limiting enzyme in 5-HT synthesis) content and 5-HT release display circadian variations within the serotonergic fibres in the SCN of rat [5]. Furthermore, TPH protein and TPH 2 mRNA (encoded by the neuronal tph gene: tph2) levels undergo circadian variations in both DR and MR [6,7]. The circadian pattern of TPH2 mRNA expression has been shown to be directly controlled by corticosterone daily surge, a neuroendocrine output of the clock [8].

While several features of serotonergic neurons have been characterized, the impact of photoperiod (the annual change in the day length) on the general functioning of these neurons is poorly investigated. Indeed, little is known about the photoperiodic-related changes in the synthesis and the release of 5-HT within the time-keeping system. For instance, the rhythmic release of 5-HT in the SCN and the IGL of golden and siberian hamsters was only investigated under long photoperiod [9-11]. On the

eISSN: 2523-6709

pISSN: 2523-6695

DOI: $10.31878 /$ ijcrpp.2020.41.01 other hand, among the studies performed on rats, the intra -SCN 5-HT release was only examined under $12 \mathrm{~h}$ light:12 h dark cycle. Furthermore, to our best knowledge, the photoperiodic regulation of the daily pattern of TPH protein and TPH 2 mRNA has never been studied.

Photoperiodic information is encoded by the SCN clock which displays photoperiod-related changes in its electrical activity $[12,13]$ and genes expression [14-18). The circadian pacemaker distributes the photoperiodic message to the target sites via several endocrine outputs. Melatonin secretion is one of these outputs and is considered as an internal message that ensures seasonal timing $[19,20]$. In addition, daily secretion of corticosterone is affected by the day-length in rats housed under short and long photoperiod [21,22] and could therefore carry photoperiodic changes to glucocorticoids sensitive neurons.

The aim of this study was to investigate whether the photoperiodic environment could influence the functioning of the serotonergic neurons in the DR and the MR. Thus, we have assessed the daily expression of TPH2 mRNA in both raphe nuclei of rats housed either under long or short photoperiod.

\section{Materials and Methods}

Animals: All experiments were performed in accordance with NIH Guidelines regarding the care of laboratory animals, with the European Communities Council Directive of 24 November 1986 (86/6.9/EEC) and the French laws.

Correspondence: Dr. Louay Labban, Faculty of Pharmacy, Al Jazeera Private University, Syria. E-Mail:1.1abban@jude.edu.sy 
Adult male Wistar rats weighing 180-200 g were purchased from the faculty of medicine in Strasbourg (France) and kept in animal's facilities for a period of one week for acclimation. Animals were then divided into two groups: the first group was placed under long photoperiod which corresponds to $18 \mathrm{~h}$ light and $6 \mathrm{~h}$ dark cycle (LP18:6, lights on from $05 \mathrm{~h} 00$ until 23h00). The second group was transferred into short photoperiod (SP6:18, lights on from $14 \mathrm{~h} 00$ until 20h00). Animals had free access to food and water during the period of this experiment. Rats in both groups were kept 4 weeks under LP or SP conditions and then killed by decapitation at seven time-points across the day/night cycle. Four animals were killed at each of the following time points: 24h00, 02h00, 04h00, 09h00, 13h00, $17 \mathrm{~h} 00$ and 21h00 for the LP group and $21 \mathrm{~h} 00,23 \mathrm{~h} 00,02 \mathrm{~h} 00,06 \mathrm{~h} 00,10 \mathrm{~h} 00$, $13 \mathrm{~h} 00$ and $18 \mathrm{~h} 00$ for the SP group. Brains were quickly removed, frozen in cold isopentane, and stored at $-80^{\circ} \mathrm{C}$ until tissue preparation. Trunk blood was collected into heparinized tubes and centrifuged $\left(2800 \mathrm{rpm}\right.$ at $\left.4^{\circ} \mathrm{C}\right)$ and the resulting serum was used for melatonin radioimmunoassay in order to confirm the integration of the photoperiodic change.

In situ hybridization procedure: Brains were cut in serial coronal sections $(20 \mu \mathrm{m}$ thick) with a cryostat (Leica Instruments $\mathrm{GmbH}$, Nussloch, Germany) throughout the rostral part of the Raphe, including DR and MR (Interaural from $+1.9 \mathrm{~mm}$ to $+0.7 \mathrm{~mm}$, Paxinos and Watson, 1982) and collected on gelatine-coated slides.

For in situ hybridization, sense and antisense riboprobes for TPH2 were prepared as described previously (Malek et al., 2005) by using the corresponding linearized plasmids and the appropriate polymerase (MAXI script, Ambion, USA $)$ in presence of [35S]-UTP $(1250 \mathrm{Ci} / \mathrm{mmol}$, Amersham, UK).

Hybridization was performed as following: sections were postfixed in $4 \%$ formaldehyde and then acetylated twice in triethanolamine $(0.1 \mathrm{M}, \mathrm{pH} 8.0)$ with $0.5 \%$ acetic anhydride. Thereafter, sections were rinsed, dehydrated in graded ethanol and air-dried. Hybridization was carried out at $54^{\circ} \mathrm{C}$ overnight in humid boxes by deposing $100 \mu \mathrm{l}$ of TPH 2 riboprobes $(300 \mathrm{pM})$. The hybridization solution contained $50 \%$ deionised formamide, $2 \mathrm{X}$ sodium saline citrate (SSC), 1X Denhardt's solution, $0.25 \mathrm{mg} / \mathrm{ml}$ yeast tRNA, $1 \mathrm{mg} / \mathrm{ml}$ salmon sperm DNA, 10\% dextran sulfate and $10 \mathrm{mM}$ dithiothreitol. After hybridization, the sections were treated with Ribonuclease A at $37^{\circ} \mathrm{C}(1.4$ $\mu \mathrm{g} / \mathrm{ml}$, Sigma, USA). Stringency washes were then performed in $4 \mathrm{X}$ SSC, $2 \mathrm{X}$ SSC, $0.5 \mathrm{X}$ SSC and $0.2 \mathrm{X}$ SSC. Finally, sections were dehydrated in graded ethanol, air-dried, and exposed for $24 \mathrm{~h}$ to an autoradiographic film (Kodak BioMax).

\section{Melatonin radioimmunoassay:}

Melatonin was extracted from plasma samples using dichloromethane and assessed in duplicates using a specific rabbit antiserum at 1:90 000 (R 19540, INRA, Nouzilly, France) and $\left[{ }^{125} \mathrm{I}\right]-2$-iodomelatonin. Melatonin assay detected values as low as $5 \mathrm{pg} / \mathrm{ml}$, and the reproducibility of the method was determined by evaluating the INTRA-assay variation $(2.5 \%)$ and the INTER-assay variation (6.5\%).

\section{Quantification procedure and statistical analysis:}

Quantitative analysis of the autoradiographic films was performed using a computerized analysis system (Biocom -program RAG 200). Total optical density (OD) was measured for each region of interest (MR, DR). The surface of each region was measured and the area of TPH2 mRNA expression was found to be comparable between the experimental groups. Non-specific OD was measured for each section within mesencephalic areas where TPH 2 mRNA is not expressed, and then subtracted from total OD. By reference to radioactive microscales, OD was converted to relative levels of mRNA. DR is divided into three distinguishable subgroups in which TPH2 mRNA levels were quantified separately: Ventromedial (VM), Dorsomedial (DM) and Lateral (LAT).

One-way analysis of variance (ANOVA I, time as factor) was conducted in order to examine the daily variation of melatonin plasma levels and TPH2 mRNA expression in the MR and all the DR subdivisions for both LP and SP animals and was followed by a Post-Hoc Newman-Keuls analysis. On the other hand, two-way analysis of variance (ANOVA II, photoperiodic condition and time as factors) and Newman-Keuls analysis were performed to compare the daily profiles of TPH 2 mRNA between LP and SP condition. For all statistical procedures, the level of significance was set at $P<0.05$.

\section{Results}

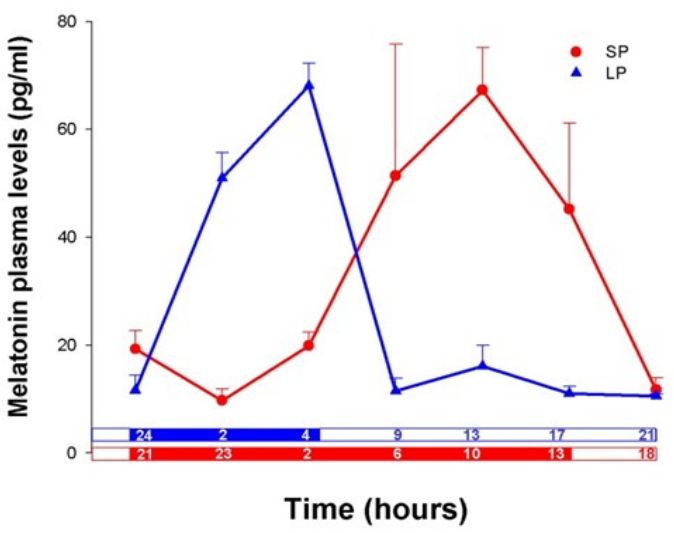

Figure 1: Daily profiles of melatonin plasma concentrations $(\mathrm{pg} / \mathrm{ml})$ measured by radioimmunoassay in rats housed under long (LP18:6, blue triangles) and short (SP6:18, red circles) photoperiods. Each symbol corresponds to the mean \pm SEM of 4 rats. The black horizontal bars represent the night period. Using ANOVA I, $* * * \mathrm{P}<0.001$ and $* * \mathrm{P}<0.01$ for the time effect on melatonin plasma levels in LP and SP rats respectively.

\section{Plasma melatonin levels}

Plasma melatonin concentrations display noticeable daily variations under both LP and SP conditions (Figure 1, $P<0.01$ for both photoperiods). The melatonin peak is delayed with the increase of the night length. Under LP, the nocturnal rise of melatonin occurs within three hours after the lights are off $(02 \mathrm{~h} 00)$ and expands on the following two hours $(04 \mathrm{~h} 00)$. The nocturnal rise under SP occurs about 10 hours after the lights are off (06h00), and lasts for the rest of the night period. These profiles are in accordance with previous results obtained in the rat and confirm the integration of the photoperiodic change. 
Effect of photoperiod on TPH2 mRNA in the MR The daily pattern of TPH2 mRNA expression in MR of rats housed under LP and SP is presented on the Figure 2. The ANOVA I analysis reveals significant daily variation of TPH2 mRNA expression in MR under both photoperiodic conditions ( $\mathrm{P}<0.05$ for both LP and SP). A sharp increase $(93 \%)$ in TPH2 mRNA levels in MR is observed at the night/day transition in LP group $(\mathrm{P}<0.05$ between $04 \mathrm{~h} 00$ and 09h00). On the other hand, a significant decrease in TPH2 mRNA levels is noticed during the night under LP (24h00/04h00: $-81 \%, \mathrm{P}<0.05)$. In all the daytime points, TPH2 mRNA expression exhibits no significant difference compared with $24 \mathrm{~h} 00$. In rats of the SP group, the maximal expression levels are also observed during the day-time $(\mathrm{P}<0.05$ between $13 \mathrm{~h} 00$ and $18 \mathrm{~h} 00)$. The percentage of the increase between these two time-points is $40 \%$. During the dark period, TPH 2 mRNA expression levels remain unchanged. Comparing the daily pattern of TPH2 mRNA in both photoperiods (ANOVA II) reveals no significant effect for time or photoperiod, though a significant interaction between both factors is observed $(\mathrm{P}<0.001)$. The latter analysis demonstrates that the daily profile of TPH 2 mRNA is different between LP and SP group.

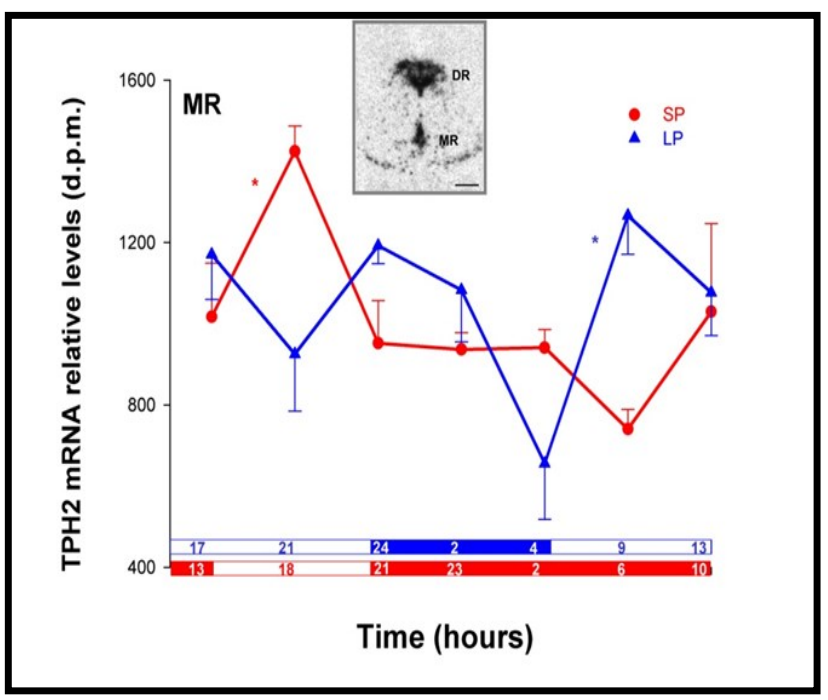

Figure 2: The daily profiles of TPH2 mRNA levels in the median raphe (MR) of rats housed under long (LP18:6, blue triangles) and short (SP6:18, red circles) photoperiods.

The top panel illustrates TPH2 mRNA in situ hybridization within the MR (scale bar: $1000 \mu \mathrm{m}$ ). Each symbol is the mean \pm SEM of 4 rats in which TPH2 mRNA was quantified throughout the caudo-rostral extent of the MR. The black horizontal bars represent the night period. ${ }^{*} \mathrm{P}<0.05$, using ANOVA I to assess the effect of the timing on the daily expression of TPH2 mRNA.

\section{Effect of photoperiod on TPH 2 mRNA in the DR}

The day/night evolution of the TPH2 mRNA expression pattern in the DR subgroups is presented in Figure 3 and is indeed quite similar to that reported in MR. TPH2 mRNA expression levels are quantified separately in each subgroup of the DR: VM, DM and LAT (Figure 3). In the $\mathrm{DR}$, the expression of TPH2 mRNA displays significant daily variations under LP $(P<0.05$ for VM, $P<0.01$ for $\mathrm{DM}$ and LAT $)$ and SP $(P<0.01$ for VM and LAT, $P<0.05$ for DM). A marked increase of TPH2 mRNA expression occurs between $04 \mathrm{~h} 00$ and $09 \mathrm{~h} 00$ under LP condition in the DR ( $90 \%$ for VM, $100 \%$ for DM and $92 \%$ for LAT, $P<0.01$ for all the subgroup). Under the same photoperiod, a significant nocturnal decrease of TPH2 mRNA levels is observed between $24 \mathrm{~h} 00$ and $04 \mathrm{~h} 00$ for VM $(-79 \%, P<0.05)$ and LAT $(-78 \%, P<0.01)$. In the $\mathrm{DM}$, however, this decrease $(-66 \%)$ fails to reach the significance limit. No significant differences are noted between $24 \mathrm{~h} 00$ and other daytime points in all DR subgroups. Under SP, the highest levels of TPH2 mRNA are reported at $18 \mathrm{~h} 00 \quad(P<0.05$ for $\mathrm{VM}$ and $\mathrm{DM}$ and $P<0.01$ for LAT, between $13 \mathrm{~h} 00$ and $18 \mathrm{~h} 00)$. The percentage of the $13 \mathrm{~h} 00 / 18 \mathrm{~h} 00$ increase is $38 \%$ for $\mathrm{VM}$, $33 \%$ for LAT and $52 \%$ for DM.

For all nighttime points, TPH2 mRNA expression levels are unchanged. The statistical comparison of TPH2 mRNA daily profiles between LP and SP condition in DR showed no significant differences in either time, except for VM $(P<0.001)$, or photoperiod. The interaction between both factors is however significant $(P<0.01$ for VM, $P<0.001$ for DM and LAT), which demonstrate that the daily pattern of TPH2 mRNA expression is different with respect to the photoperiod.

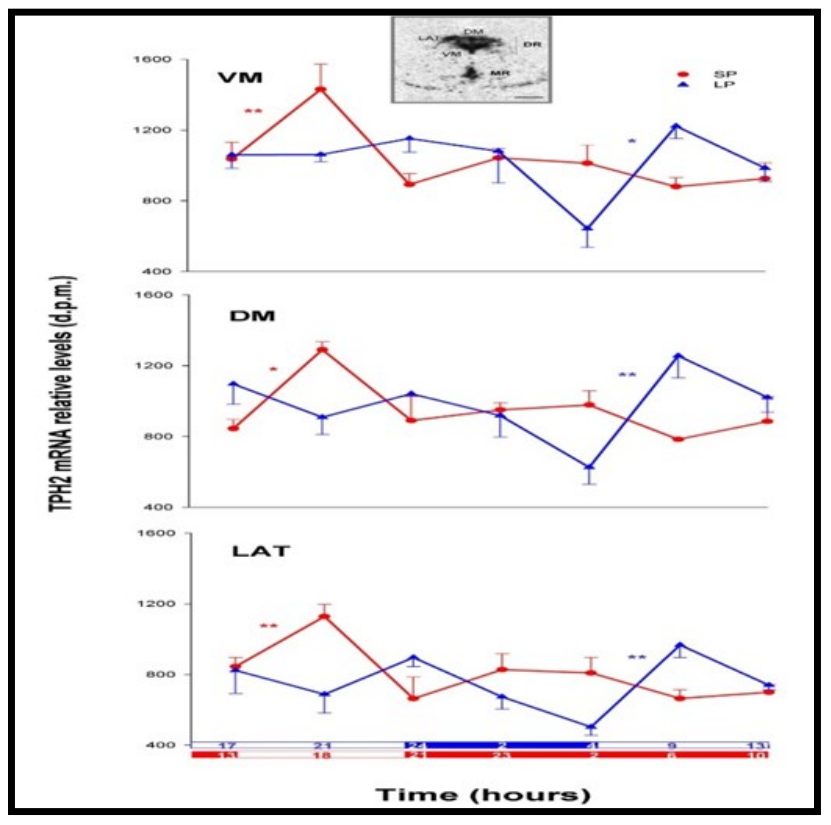

Figure 3: TPH2 mRNA relative levels

Dorsal raphe (DR) subdivisions; ventromedian (VM), dorsomedian (DM) and lateral (LAT) of rats housed under long (LP18:6, blue triangles) and short (SP6:18, red circles) photoperiods. The anatomical subgroups of the DR are illustrated using TPH2 mRNA in situ hybridisation (the top panel, scale bar: $1000 \mu \mathrm{m}$ ). Each symbol is the mean $\square$ SEM of 4 rats in which TPH2 mRNA was quantified throughout the caudo-rostral extent of each subdivision. The black horizontal bars represent the night period. ${ }^{*} \mathrm{P}<0.05$ and $* * \mathrm{P}<0.01$, using ANOVA I to assess the effect of the timing on the daily expression of TPH 2 mRNA.

\section{Discussion}

To our knowledge, this is the first evidence for photoperiod-related changes in the daily pattern of the tph2 gene expression in rats. Under standard lighting conditions (i.e. LD12:12), TPH2 mRNA undergoes rhythmic expression and the higher levels are observed 2 hours before the day/night transition in both raphe nuclei 
(7). In this study, we report a similar expression pattern under SP8:16, where TPH2 mRNA levels peak at 18h00 (lights off at 20h00) in both MR and DR. This is however different to that in LP group, for which TPH2 mRNA levels peak at the beginning of the light period and are unaffected by the day/night transition. Under both photoperiodic conditions, TPH2 mRNA levels increase with the lights on, which might reflect an effect of the light onset on its expression. Previous tract tracing studies have described the presence of retinal input to the DR, suggesting that 5-HT neurons can receive the light/dark information $[23,24]$.

The effect of light on TPH 2 mRNA expression needs to be further investigated. It is noteworthy that the daily pattern of TPH 2 mRNA under LP tends to be bimodal because the expression levels display a marked nocturnal decrease in DR and MR before the peak at the beginning of the light period. This bimodal pattern is, however not evident in VM and DM, and the nocturnal decrease of TPH2 mRNA levels in the latter subgroup is not significant. A bimodal pattern in serotonergic neurones has been previously reported in rats under LD12:12 conditions concerning 5-HT content in the raphe [25] as well as TPH protein content in the SCN (5). However, in the latter study, the bimodal pattern was not found in the daily profile of 5-HT release in the SCN, which displays a single peak at the beginning of the dark period.

In our previous studies, we have described a temporal relationship between TPH2 mRNA, TPH protein and 5HT release in the rat circadian system under standard lighting conditions. Indeed, these three parameters display rhythmic and time-related variations under LD12:12 cycle. The circadian variations of TPH 2 mRNA in the DR and MR are found at the protein level in the same nuclei, as well as in the 5-HT terminals within the SCN and the IGL [5-7]. Furthermore, the 5-HT release is rhythmic in the SCN [5] and the IGL [11]. Herein, we demonstrate a daily differential pattern of TPH2 mRNA expression in both DR and MR concerning photoperiodic environment. It could be suggested that the rhythmic patterns of TPH protein levels and 5-HT release would display photoperiod-related changes within both RD $\square$ IGL and RM $\square$ SCN pathways. Further experiments should be done in order to confirm such hypothesis.

We have previously demonstrated that TPH2 mRNA expression under constant darkness condition displays significant variations that are similar to those observed under LD12:12, indicating a circadian regulation of this expression [6]. This later result confirms that TPH2 mRNA daily pattern is under the SCN clock control. Indeed, under LD12:12, the rhythmic expression of TPH2 mRNA has been shown to be controlled by glucocorticoids, one of the endocrine outputs of the SCN central pacemaker [26-28]

The abolishment of corticosterone surge by adrenalectomy results in a complete suppression of TPH 2 mRNA rhythm, which can be reinstated using a paradigm of rhythmic replacement of corticosterone [7]. In rats, the daily secretion of corticosterone is affected by the daylength $[9,10]$ and could thereby provide an internal signal not only for the circadian time but also for the seasonal time. Glucocorticoids might thus drive the photoperiodrelated changes in TPH 2 mRNA daily rhythm described in the present study.
To further confirm the involvement of corticosterone, it would be relevant to examine the effect of photoperiodic restitution of corticosterone on the TPH2 mRNA daily expression in adrenalectomised rats housed under both photoperiodic regimens. Furthermore, we can investigate the influence of photoperiodic changes upon GR expression levels within both DR and MR, to assess whether the sensitivity of the serotonergic neurons to corticosterone is modified according to the photoperiod.

Melatonin secretion is a seasonal output of the $\mathrm{SCN}$ known to drive photoperiodic message. Indeed, the duration of the nocturnal melatonin secretion is proportional to the length of darkness, providing an internal signal for seasonal changes [28]. A primary target tissue of this seasonal signal in mammals is the hypophyseal pars tuberalis in which melatonin is a key regulator of clock genes expression [26-29].

Melatonin could also be involved, as a seasonal signal, in the photoperiodic changes of TPH 2 mRNA daily rhythm reported in the present study. Although melatonin receptors have not been identified in the raphe neurons [30], this hormone has been shown to modulate 5-HT synthesis in the SCN [31]. It can be suggested that melatonin would act indirectly upon the serotonergic neurones via melatonin-sensitive brain areas. Further experiments are needed to investigate the influence of melatonin on the photoperiodic related changes of TPH2 mRNA rhythmic expression.

\section{Conclusion}

Raphe serotonergic neurons are sensitive to changes in the photoperiodic environment. The daily pattern of TPH2 mRNA is affected by these changes, which might influence TPH protein levels and 5-HT release within the $\mathrm{SCN}$. If so, the involvement of the serotonergic system in the photoperiodic time measurement should be further considered. Moreover, photoperiodic changes in 5-HT release within the SCN, and elsewhere in the brain, would reflect the involvement of the serotonergic system in seasonal affective disorder.

\section{Source of funding: Self}

Conflict of Interest: Nil

\section{References}

[1] Charles M. Morin. Behavioral and Pharmacological Therapies for Late-Life InsomniaA Randomized Controlled Trial. JAMA. 1999;281(11):991-9.

[2] Yannielli PC, Brewer JM, Harrington ME. Blockade of the NPY Y5 receptor potentiates circadian responses to light: complementary in vivo and in vitro studies. Eur J Neurosci. 2004;19 (4):891-7.

[3] Meyer-Bernstein EL, Morin LP. Differential serotonergic innervation of the suprachiasmatic nucleus and the intergeniculate leaflet and its role in circadian rhythm modulation. J Neurosci. 1996;16(6):2097-111.

[4] Hay-Schmidt. Projections from the raphe nuclei to the suprachiasmatic nucleus of the rat. Journal of Chemical Neuroanatomy. 2003;25(4):293-310 
[5] Barassin. Circadian tryptophan hydroxylase levels and serotonin release in the suprachiasmatic nucleus of the rat. The European journal of neuroscience. 2002;15. 833-40.

[6] Malek ZS, Pevet P, Raison S. Circadian changes in Tryptophan Hydroxylase protein levels within the Rat Intergeniculate Leaflets and Raphe Nuclei. Neuroscience 2004;125(3): 749-58.

[7] Malek ZS. Tissue-specific expression of tryptophan hydroxylase mRNAs in the rat midbrain: anatomical evidence and daily profiles European Journal of Neurosciences. 2005;22 (4):895-901.

[8] Zeina S. Malek, Dominique Sage, Paul Pévet, Sylvie Raison, Daily Rhythm of Tryptophan Hydroxylase-2 Messenger Ribonucleic Acid within Raphe Neurons Is Induced by Corticoid Daily Surge and Modulated by Enhanced Locomotor Activity, Endocrinology. 2007; 148 (11): 5165-72

[9] Glass. Serotonin Modulates Photic Responses in the Hamster Suprachiasmatic Nuclei. The Journal of Neuroscience.1994;74(6): 3635-42

[10] Dudley TE, DiNardo LA, Glass JD. Endogenous regulation of serotonin release in the hamster suprachiasmatic nucleus. J Neurosci. 1998;18 (13):5045-52

[11] Grossman. Human T Regulatory Cells Can Usethe Perforin Pathway to Cause Autologous Target Cell Death. Immunity. 2004;21: 589-6001,

[12] Mrugala. Rhythmic multiunit neural activity in slices of hamstersuprachiasmatic nucleus reflect prior photoperiod. Am J Physiol Regulatory Integrative Comp Physiol. 2000;278: R987-94

[13] Vanderleest. Seasonal encoding by the circadian pacemaker of the SCN. Curr Biol. 2007;17(5):46873

[14] Sumová. The rat suprachiasmatic nucleus is a clock for all seasons. Proceedings of the National Academy of Sciences of the United States of America. 1995;92:7754-8.

[15] Tournier. Photoperiod differentially regulates clock genes' expression in the suprachiasmatic nucleus of Syrian hamster. Neuroscience. 2003;118(2):317-322

[16] Johnston JD, Ebling FJ, Hazlerigg DG. Photoperiod regulates multiple gene expression in the suprachiasmatic nuclei and pars tuberalis of the Siberian hamster (Phodopus sungorus).Eur J Neurosci. 2005;21(11):2967-74

[17] Oster H, Koch CE, Leinweber B, Drengberg BC, Blaum C. Interaction between circadian rhythms and stress. Neurobiology of Stress. 2017;6:57-67

[18] Bering T, Hertz H, Rath MF. Rhythmic release of corticosterone induces circadian clock gene expression in the cerebellum. Neuroendocrinology. 2019; doi: 10.1159/000503720
[19] Simonneaux, V. and Ribelayga, C. Generation of the melatonin endocrine message in mammals: a review of the complex regulation of melatonin synthesis by norepinephrine, peptides, and other pineal transmitters. Pharmacol Rev. 2003;55 (2):325-95.

[20] Ahlersova E, Ahlers I, and Smajda B. Influence of light regimen and time of year on circadian oscillations of insulin and corticosterone in rats. Physiological research / Academia Scientiarum Bohemoslovaca. February 1992;41(4):307-14

[21] Illnerova H, Hoffman K, Vaněcek J. Adjustment ofthe rat pineal Nacetyltransferase rhythm to change fromlong to short photoperiod depends on the direction of theextension of the dark period. Brain Res . 1986;362:403-8.

[22] Christophe Ribelayga, $\mathrm{Yu} \mathrm{Cao,} \mathrm{and} \mathrm{Stuart} \mathrm{C.}$ Mangel. The Circadian Clock in the Retina Controls Rod-Cone Coupling. Neuron. 2008;59 (5):790-801

[23] Shen \& Semba. A direct retinal projection to the dorsal raphe nucleus in the rat. Brain Res. 1994;635(1-2):159-68.

[24] Fite KV, Janusonis S, Foote W, Bengston L. Retinal afferents tothe dorsal raphe nucleus in rats and Mongolian gerbils. J CompNeurol. (1999) 414:469-484.

[25] Pinato. Bimodal Daily Variation in the Serotonin Content in theRaphe Nuclei of Rats. Biological Rhythm Research. 2004; 0165-0424,

[26] Messager S, Ross AW, Barrett P, and Morgan PJ. Decoding photoperiodic time through Perl and ICER gene amplitude. PNAS.1999; 96 (17): 993843

[27] Dardente et al. Melatonin inducesCrylexpression in the pars tuberalis of the rat. Molecular Brain Research. 2003;114: 101-6.

[28] Johnston JD1, Tournier BB, Andersson H, Masson -Pévet M, Lincoln GA, Hazlerigg DG. Multiple effects of melatonin on rhythmic clock gene expression in the mammalian pars tuberalis. Endocrinology. 2006;147(2):959-65

[29] Wagner GC, Johnston JD, Tournier BB, Ebling FJ, Hazlerigg DG. Melatonin induces gene-specific effects on rhythmic mRNA expression in the pars tuberalis of the Siberian hamster (Phodopus sungorus). Eur J Neurosci. 2007;25(2):485-90.

[30] Gauer F, Masson-Pevet M, Stehle J, and Pevet P. Daily variations in melatonin receptor density of rat pars tuberalis and suprachiasmatic nuclei are distinctly regulated. Brain Research. 1994;641(1): 92-8.

[31] Recio J, Pévet P, Vivien-Roels B, Míguez JM, Masson-Pévet M.. Daily and Photoperiodic Melatonin Binding Changes in the Suprachiasmatic Nuclei, Paraventricular Thalamic Nuclei, and Pars Tuberalis of the Female Siberian Hamster (Phodopus sungorus). Journal of Biological rhythms. 1996;11(4):325-32. 